\title{
Arithmetic progressions with common difference divisible by small primes
}

by

\author{
N. Saradha (Mumbai) and R. TiJdeman (Leiden)
}

1. Introduction. For any integer $n \geq 1$ let $P(n)$ and $p(n)$ denote the greatest prime factor and smallest prime factor of $n$, respectively. Also let $P(1)=p(1)=1$. We consider the equation

$$
n(n+d) \cdots(n+(k-1) d)=b y^{l}
$$

in positive integers $n, k \geq 2, d>1, b, y, l \geq 3$ with $l$ prime, $\operatorname{gcd}(n, d)=1$ and $P(b) \leq k$. We write

$$
d=D_{1} D_{2},
$$

where $D_{1}$ is the maximal divisor of $d$ such that all prime divisors of $D_{1}$ are congruent to $1(\bmod l)$. Thus $D_{1}$ and $D_{2}$ are relatively prime positive integers such that $D_{2}$ has no prime divisor which is congruent to $1(\bmod l)$. Shorey [Sh88] proved that (1.1) implies

$$
D_{1}>1 \quad \text { if } k \geq C_{1},
$$

where $C_{1}$ is a large absolute constant. In [SS01], Saradha and Shorey showed that $C_{1}=4$ suffices. Thus for all $k \geq 4$, there exists a prime $\equiv 1(\bmod l)$ dividing $d$. Since $l \geq 3$, this implies that (1.1) has no solution if $d$ is composed of the primes 2, 3, and 5 only. For $k=3$, Györy [G99] showed that (1.1) with $P(b)<k$ is impossible. Further, from [SS01], it follows that (1.3) holds for (1.1) when $k=3$ provided 2 or 3 divides $d$. Shorey and Tijdeman [ST90] sharpened (1.3) to

$$
D_{1}>C_{2} k^{l-2} .
$$

The constant $C_{2}$ turns out to be very small and therefore the above inequality is trivial for small values of $k$.

2000 Mathematics Subject Classification: Primary 11D61.

Key words and phrases: arithmetic progression, perfect powers, multiplicative covering. 
In [SS01], estimates for $D_{1}$ which were non-trivial even for small values of $k$ were given. For example, it was shown that

$$
D_{1}>1.59 \theta k^{l / 2-3.15} \quad \text { for } l \geq 17,
$$

where

$$
\theta= \begin{cases}1 & \text { if } l \nmid d \\ 1 / l & \text { if } l \mid d\end{cases}
$$

The reduction in the exponent of $k$ from $l-2$ in (1.4) to $l / 2-3.15$ in (1.5) is due to using a counting argument of Erdős and Selfridge while covering small values of $k$ (see [SS01, Lemma 9]). When $k \geq 11380$, it was shown in [SS01, Lemma 7] that

$$
D_{1}>\theta k^{l-3+1 / l} \text {. }
$$

The proof of this inequality depends on a graph-theoretic argument due to Erdős and Selfridge [ES75] and some further refinements in [Sa97]. In this paper, we improve this graph-theoretic argument (see Lemma 4.2). Using this improvement we show

Theorem 1.1. Let (1.1) hold with $l \geq 5$. Put

$$
E_{1}=\max \left(0.7 \theta k^{l-3}, \frac{l \theta}{2 k} n^{(l-2) / l}\right), \quad E_{2}=\max \left(0.7 \theta k^{l-4}, \frac{l \theta}{3 k} n^{(l-3) / l}\right) .
$$

(i) Suppose $k \geq 4$ and $d$ is divisible by 2 or 3 . Then

$$
D_{1}>E_{1} \text {. }
$$

(ii) Suppose $5 \mid d$. Then

$$
D_{1}>E_{1} \quad \text { if } k \geq 8 \text { or } k=6 \text { and } D_{1}>E_{2} \quad \text { if } k=7 .
$$

(iii) Suppose $7 \mid d$. Then

$$
D_{1}>E_{1} \quad \text { if } k \geq 25 \text { and } D_{1}>E_{2} \quad \text { if } 8 \leq k \leq 24 .
$$

In [BBGH06], it was shown that (1.1) with $4 \leq k \leq 11$ and $P(b) \leq$ $k / 2$ has no solution. This result depends on Galois representation theory of modular forms. As an immediate consequence of this result and Theorem 1.1 we get the following corollary.

Corollary 1.2. Let (1.1) hold with $k \geq 4, P(b) \leq k / 2$ and $l \geq 5$. Then

(i) $D_{1}>E_{1}$ if 2 or 3 or 5 divides $d$.

(ii) $D_{1}>E_{2}$ if $7 \mid d$.

Remarks. (i) When $l=3$, it was shown in [SS01, Theorem 3] that

$$
D_{1}>0.41 \theta k^{1 / 3} \text {. }
$$

We do not have any improvement over this. 
(ii) Let $k=3$. As mentioned earlier, (1.1) with $P(b)<3$ does not hold. Now let $P(b)=3$. Suppose $2 \mid d$. Then $n(n+d)(n+2 d)=3^{\alpha} y^{l}$ for some integer $\alpha>0$. Hence $(n, n+d, n+2 d)=\left(3^{\alpha} y_{1}^{l}, y_{2}^{l}, y_{3}^{l}\right)$ or $\left(y_{1}^{l}, 3^{\alpha} y_{2}^{l}, y_{3}^{l}\right)$ or $\left(y_{1}^{l}, y_{2}^{l}, 3^{\alpha} y_{3}^{l}\right)$ for some positive integers $y_{1}, y_{2}$ and $y_{3}$. Thus

$$
y_{3}^{l}-y_{2}^{l}=d \quad \text { or } \quad y_{3}^{l}-y_{1}^{l}=2 d \quad \text { or } \quad y_{2}^{l}-y_{1}^{l}=d .
$$

Now we see that (1.3) holds since the difference of two $l$ th powers is always divisible by a prime congruent to $1(\bmod l)$. Note that $3 \nmid d \operatorname{since} \operatorname{gcd}(n, d)$ $=1$. It is still not known if (1.3) holds in the remaining case of $d$ odd and $3 \nmid d$.

(iii) The constant 0.7 in the definitions of $E_{1}$ and $E_{2}$ is obtained from [SS01, Lemma 5] by taking $\kappa=7, l \geq 5$ and $l^{\prime}=2,3$.

\section{Basic lemmas}

Lemma 2.1 ([SS01, Lemma 1]). For $0 \leq i<k$, let $n+i d=a_{i} a_{i}^{\prime}$, where $a_{i}$ is a positive integer with $P\left(a_{i}\right) \leq k$ for $0 \leq i<k$. Let $S=\left\{a_{0}, \ldots, a_{k-1}\right\}$. For every prime $p \leq k$ with $\operatorname{gcd}(p, d)=1$, choose $a_{i_{p}} \in S$ such that $p$ does not appear to a higher power in the factorization of any other element of $S$. Let $S_{1}$ be the subset of $S$ obtained by deleting from $S$ all $a_{i_{p}}$ with $p \leq k$ and $\operatorname{gcd}(p, d)=1$. Then

$$
\prod_{a_{i} \in S_{1}} a_{i} \leq(k-1) ! \prod_{p \mid d} p^{-\operatorname{ord}_{p}(k-1) !} .
$$

Next we combine [SS05, Lemma 10] and [SS01, Lemma 5] to get

Lemma 2.2. Assume that (1.1) holds.

(i) If

$$
D_{1} \leq \min \left(0.7 \theta k^{l-3}, \frac{l \theta}{2 k} n^{(l-2) / l}\right),
$$

then the products $a_{i_{1}} a_{i_{2}}$ with $0 \leq i_{1} \leq i_{2}<k$ are all distinct.

(ii) If

$$
D_{1} \leq \min \left(0.7 \theta k^{l-4}, \frac{l \theta}{3 k} n^{(l-3) / l}\right),
$$

then the products $a_{i_{1}} a_{i_{2}} a_{i_{3}}$ with $0 \leq i_{1} \leq i_{2} \leq i_{3}<k$ are all distinct.

We assume (2.2) or (2.3) according to the situation we consider. Under these assumptions $a_{i}$ 's are distinct.

We need to count the number of $a_{i}$ 's composed of certain primes. Several counting functions have been used earlier. See [Sa97], [SS01] and [SS05]. Let $2=p_{1}<p_{2}<\cdots$ be the sequence of all primes and $q_{1}<q_{2}<\cdots$ be the sequence of primes coprime to $d$. Let $\pi(k)$ and $\pi_{d}(k)$ denote the number of primes $\leq k$ and the number of primes $\leq k$ which are coprime to $d$, 
respectively. Let $C\left(k, m, \alpha_{1}, \ldots, \alpha_{m}, r_{1}, \ldots, r_{h}\right)$ denote the number of $a_{r}$ 's not divisible by $q_{i}^{\alpha_{i}+1}$ for $1 \leq i \leq m$, not divisible by the primes $q_{m+1}, \ldots$, and not by certain integers $r_{1}, \ldots, r_{h}$. Obviously,

$$
\begin{aligned}
& C\left(k, m, \alpha_{1}, \ldots, \alpha_{m}, r_{1}, \ldots, r_{h}\right) \\
& \geq k-\sum_{i=1}^{m}\left\lceil\frac{k}{q_{i}^{\alpha_{i}+1}}\right\rceil-\sum_{q_{m}<p \leq k}\left\lceil\frac{k}{p}\right\rceil-\sum_{s=1}^{h}\left\lceil\frac{k}{r_{s}}\right\rceil,
\end{aligned}
$$

where $\lceil x\rceil$ denotes the smallest integer greater than or equal to $x$. For $h=0$, we take the last sum to be 0 and write the function as $C\left(k, m, \alpha_{1}, \ldots, \alpha_{m}\right)$.

3. Sets with distinct products. For any set $S$, by $a S$ we mean the set $\{a x \mid x \in S\}$. We say that $S$ has property $P_{i}$ if the products $x_{1} \cdots x_{i}$ are all distinct for any $i$-tuple $x_{1} \leq \cdots \leq x_{i}$ with $x_{j} \in S$ for $1 \leq j \leq i$. If $S$ has property $P_{2}$, the products $x y$ with $x \leq y, x, y \in S$, are all distinct. We observe that if $S$ has property $P_{i}$ for some $i \geq 2$, then $S$ has property $P_{j}$ for any $j \leq i$. Suppose (1.1) holds with (2.2); then the set of $a_{i}$ 's has property $P_{2}$, by Lemma 2.2 .

LEMmA 3.1. Let $X \subseteq\left\{1, a, \ldots, a^{r}\right\}$ with $r \leq 5$ and let $n_{1}, \beta_{1}, \ldots, \beta_{n_{1}}$ be positive integers with

$$
Y=\bigcup_{i=1}^{n_{1}} \beta_{i} X
$$

Let $S \subseteq Y$ be any subset of $Y$ having property $P_{2}$. Let $S_{i}=\beta_{i} X \cap S$ for $i=1, \ldots, n_{1}$ and assume $\left|S_{1}\right| \geq\left|S_{2}\right| \geq \cdots$. Then

$$
|S| \leq \begin{cases}\min \left\{2 n_{1}+1, n_{1}+r-1\right\} & \text { if }\left|S_{1}\right|=3 \\ \min \left\{2 n_{1}, n_{1}+r\right\} & \text { if }\left|S_{1}\right|=2 .\end{cases}
$$

Proof. Let $1 \leq i \leq n_{1}$. Let $t_{i}$ be the least non-negative integer such that

$$
a^{t_{i}} \beta_{i} \in S_{i} \text {. }
$$

Put $\gamma_{i}=a^{t_{i}} \beta_{i}$. Then $S_{i} \subseteq \gamma_{i}\left\{1, a, \ldots, a^{5}\right\}$ and $\gamma_{i} \in S_{i}$. Since $S$ has property $P_{2}$, each $S_{i}$ has property $P_{2}$. Observe that all the differences of the exponents of $a$ of pairs of elements from some $S_{i}$ have to be distinct, i.e., there are no non-negative integers $x_{1}<y_{1}$ and $x_{2}<y_{2}$ with

$$
\gamma_{i_{1}} a^{x_{1}}, \gamma_{i_{1}} a^{y_{1}} \in S_{i_{1}}, \quad \gamma_{i_{2}} a^{x_{2}}, \gamma_{i_{2}} a^{y_{2}} \in S_{i_{2}} \quad \text { and } \quad y_{1}-x_{1}=y_{2}-x_{2}
$$

for some $i_{1}$ and $i_{2}$ with $1 \leq i_{1}, i_{2} \leq n_{1}$. This is because if (3.2) holds, then

$$
\gamma_{i_{1}} a^{x_{1}} \cdot \gamma_{i_{2}} a^{y_{2}}=\gamma_{i_{1}} a^{y_{1}} \cdot \gamma_{i_{2}} a^{x_{2}}
$$

contradicting property $P_{2}$. As $S \subseteq\left\{1, a, a^{2}, a^{3}, a^{4}, a^{5}\right\}$, only the five differences $1,2,3,4,5$ are available. Observe that if $\left|S_{1}\right|=4$ it generates 6 differences, and if $\left|S_{1}\right|=3$ then 3 differences. Hence we obtain $\left|S_{1}\right| \leq 3$ and $\left|S_{i}\right| \leq 2$ for $i>1$. Thus $|S| \leq 2 n_{1}+1$ if $\left|S_{1}\right|=3$ and $|S| \leq 2 n_{1}$ if $\left|S_{1}\right| \leq 2$. 
Moreover, if $S=\left\{1, a, a^{2}, \ldots, a^{r}\right\}$, then the number of sets $S_{i}$ with $\left|S_{i}\right|=2$ is at most $r-3$ if $\left|S_{1}\right|=3$ and at most $r$ if $\left|S_{1}\right|=2$. Thus

$$
|S| \leq 3+2(r-3)+\left(n_{1}-r+2\right)=n_{1}+r-1
$$

if $\left|S_{1}\right|=3$, and otherwise

$$
|S| \leq 2 r+n_{1}-r=n_{1}+r
$$

Lemma 3.2. Let $X \subseteq\left\{1, a, \ldots, a^{r}\right\}$ with $r \leq 5$ and let $n_{1}, \beta_{1}, \ldots, \beta_{n_{1}}$ be positive integers with

$$
Y=\bigcup_{i=1}^{n_{1}} \beta_{i} X
$$

Let $S \subseteq Y$ be any subset of $Y$ having property $P_{3}$. Let $S_{i}=\beta_{i} X \cap S$ for $i=1, \ldots, n_{1}$ and assume $\left|S_{1}\right| \geq\left|S_{2}\right| \geq \cdots$. Then

$$
|S| \leq \begin{cases}n_{1}+3 & \text { if } X \subseteq\left\{1, a, a^{2}, a^{3}, a^{4}, a^{5}\right\}, \\ n_{1}+2 & \text { if } X \subseteq\left\{1, a, a^{2}, a^{3}, a^{4}\right\}, \\ n_{1}+1 & \text { if } X \subseteq\left\{1, a, a^{2}\right\} .\end{cases}
$$

Proof. As seen in Lemma 3.1, there exists $\gamma_{i}$ such that $S_{i} \subseteq \gamma_{i}\{1, a$, $\left.\ldots, a^{5}\right\}$ and $\gamma_{i} \in S_{i}$ and

$$
\left|S_{1}\right| \leq 3 \quad \text { and } \quad\left|S_{i}\right| \leq 2 \text { for } i>1 .
$$

Also, there are no positive integers $x_{1}, y_{1}$ and $x_{2}, y_{2}$ for which (3.2) holds for any $i_{1}, i_{2}$ with $1 \leq i_{1}, i_{2} \leq n_{1}$. Further, property $P_{3}$ implies that there are no positive integers $x, y$ and $z$ with $\gamma_{i_{1}} a^{x} \in S_{i_{1}}, \gamma_{i_{2}} a^{y} \in S_{i_{2}}, \gamma_{i_{3}} a^{z} \in S_{i_{3}}$ for some $i_{1}, i_{2}, i_{3}$ with $1 \leq i_{1}, i_{2}, i_{3} \leq n_{1}$ such that

$$
x+y=z \quad \text { or } \quad x=2 y .
$$

Suppose the first possibility occurs; then

$$
\left(\gamma_{i_{1}} a^{x}\right)\left(\gamma_{i_{2}} a^{y}\right)\left(\gamma_{i_{3}}\right)=\left(\gamma_{i_{1}}\right)\left(\gamma_{i_{2}}\right)\left(\gamma_{i_{3}} a^{z}\right),
$$

contradicting $P_{3}$. Suppose the second possibility occurs; then,

$$
\left(\gamma_{i_{1}} a^{x}\right)\left(\gamma_{i_{2}}\right)^{2}=\left(\gamma_{i_{1}}\right)\left(\gamma_{i_{2}} a^{y}\right)^{2}
$$

again contradicting $P_{3}$. Using the above observations we find that if $\left|S_{1}\right|=3$, then $\left|S_{i}\right| \leq 1$ for $i \geq 2$, giving $|S| \leq n_{1}+2$. This can only happen if $r>2$. Let $\left|S_{1}\right|=2$. In this case if $X \subseteq\left\{1, a, a^{2}, a^{3}, a^{4}\right\}$, then $\left|S_{i}\right| \leq 1$ for $i \geq 2$. If $X \subseteq\left\{1, a, a^{2}, a^{3}, a^{4}, a^{5}\right\}$, then $\left|S_{3}\right| \leq\left|S_{2}\right| \leq 2$ and $\left|S_{i}\right| \leq 1$ for $i \geq 4$. The lemma follows.

4. Lemmas based on graph theory. Let $X \geq 1$ and $S \subseteq[1, X]$ be a set of integers. Let $U$ and $V$ be such that every integer in $S$ can be expressed as $u v$ with $u \in U$ and $v \in V$. We call such a pair of sets $(U, V)$ a multiplicative covering for $S$. This construction was first given in [ES75] 
when $S=[1, X]$ and it was refined in [Sa97, p. 157]. Let $i \geq 1$ be an integer. In the lemma below we construct a multiplicative covering $(U, V)$ for a set $S$ of integers not divisible by some given prime.

Lemma 4.1. Let $i \geq 1$ be an integer and $S$ be the set of positive integers $\leq X$ not divisible by $p_{i}$. Take integers $m \geq 1$ and $T \geq 1$. Let $U=U(m, T)$ denote the set of integers $<T$ composed of $p_{1}, \ldots, p_{m}$ and not divisible by $p_{i}$. With every prime $p_{j}, j \neq i$, let the integer $r_{j}(T)$ denote the smallest integer $\geq T$ not divisible by $p_{i}$ with $P\left(r_{j}(T)\right)=p_{j}$. Define

$$
\begin{aligned}
V_{j} & =\left\{w \mid w \leq p_{j} X / r_{j}(T), p(w)=p_{j} \text { and } p_{i} \nmid w \text { for } 1 \leq j \leq m\right\}, \\
V_{m+1} & =\left\{w \mid w \leq X, w=1 \text { or } p(w) \geq p_{m+1} \text { and } p_{i} \nmid w\right\} .
\end{aligned}
$$

Put

$$
V=\bigcup_{j=1}^{m+1} V_{j}
$$

(Note that $V_{i}=\emptyset$ if $i \leq m$.) Then

$$
|V|=\sum_{j=1, j \neq i}^{m+1}\left(\frac{\varphi\left(p_{1} \cdots p_{j-1} p_{i}^{(j)}\right)}{p_{1} \cdots p_{j-1} p_{i}^{(j)}} \frac{X}{r_{j}(T)}+E_{j}\right)
$$

where for $1 \leq j \leq m+1, j \neq i$, we define

$$
p_{i}^{(j)}= \begin{cases}p_{i} & \text { if } j<i \leq m \text { or } m<i \\ 1 & \text { otherwise }\end{cases}
$$

and

$$
E_{j} \leq \max \left\{\varrho(z)-\frac{\varphi\left(p_{1} \cdots p_{j-1} p_{i}^{(j)}\right) z}{p_{1} \cdots p_{j-1} p_{i}^{(j)}}\right\},
$$

where $\varrho(z)$ is the number of integers $\leq z$ and coprime to $p_{1}, \ldots, p_{j-1}, p_{i}^{(j)}$ and the maximum is taken over all $z$ with $0 \leq z<p_{1} \cdots p_{j-1} p_{i}^{(j)}$ and $\operatorname{gcd}\left(z, p_{1} \cdots p_{j-1} p_{i}^{(j)}\right)=1$.

We refer to [Sa97] for the above construction. The fact that such a pair $(U, V)$ is a multiplicative covering for $S$ can be easily checked.

The following is a refinement of Lemma 3 of [ES75] which depends on graph theory. Let $R$ be a given set of integers having property $P_{2}$, i.e. all products $r_{1} r_{2}$ with $r_{1} \leq r_{2}$ and $r_{1}, r_{2} \in R$ are distinct. Let $(U, V)$ be a multiplicative covering for $[1, X]$. We draw a bipartite graph $G_{R}=G_{R}(U, V)$ as follows. The vertices of the bipartite graph are the integers in $U$ and the integers in $V$. We draw an edge between a vertex $u \in U$ and a vertex $v \in V$ if $u v$ equals an integer $r \in R$. Since $R$ satisfies $P_{2}$, the graph $G_{R}$ contains no rectangle. In [ES75], it was shown that $E_{R}$, the number of edges in $G_{R}$, 
satisfies

$$
E_{R} \leq|V|+\left(\begin{array}{c}
|U| \\
2
\end{array}\right)
$$

We improve the inequality as follows.

LEMMA 4.2. Let $R$ be a set of integers having property $P_{2}$. Let $G_{R}$ be the graph drawn as above. Then

$$
E_{R} \leq|V|+|W(U)|
$$

where $W(U)$ is the set of ratios $>1$ of pairs of integers from $U$.

REMARK 4.3. Obviously we have $|W(U)| \leq\left(\begin{array}{c}|U| \\ 2\end{array}\right)$, but in our applications $|W(U)|$ is much smaller than $\left(\begin{array}{c}|U| \\ 2\end{array}\right)$.

REMARK 4.4. By using Lemma 3 of [ES75], it has been shown in [Sa97] that (1.1) implies that $k \leq 11380$ as compared to $\leq 30000$ obtained in [ES75]. It is clear that the improvement obtained in Lemma 4.2 will further reduce the bound for $k$.

Proof of Lemma 4.2. We follow the proof of [ES75]. If a pair of edges emanate from the same vertex, we call the pair a concurrent pair. For $i \geq 1$, let $s_{i}$ denote the number of vertices in $V$ from which $i$ edges emanate. Then

$$
E_{R}=\sum_{i \geq 1} i s_{i}=\sum_{i \geq 1} s_{i}+\sum_{i \geq 2}(i-1) s_{i} \leq|V|+\sum_{i \geq 2}\left(\begin{array}{l}
i \\
2
\end{array}\right) s_{i} .
$$

Let us consider a vertex $v \in V$ from which $i$ edges emanate. The number of concurrent pairs is $\left(\begin{array}{l}i \\ 2\end{array}\right)$. Thus the total number of concurrent pairs in the graph is

$$
\sum_{i \geq 2}\left(\begin{array}{l}
i \\
2
\end{array}\right) s_{i}
$$

Let $u_{1}, u_{1}^{\prime}, u_{2}, u_{2}^{\prime}$ be elements of $U$ such that

$$
\frac{u_{1}^{\prime}}{u_{1}}=\frac{u_{2}^{\prime}}{u_{2}}
$$

Suppose $u_{1}$ and $u_{1}^{\prime}$ are the end points of a concurrent pair of edges, as also are $u_{2}$ and $u_{2}^{\prime}$. Then there exist $v_{1}, v_{2} \in V$ such that

$$
u_{1} v_{1}=r_{1}, \quad u_{1}^{\prime} v_{1}=r_{2}, \quad u_{2} v_{2}=r_{3}, \quad u_{2}^{\prime} v_{2}=r_{4}
$$

with $r_{1}, r_{2}, r_{3}, r_{4} \in R$. Hence

$$
r_{1} r_{4}=u_{1} v_{1} u_{2}^{\prime} v_{2}=u_{1}^{\prime} u_{2} v_{1} v_{2}=r_{2} r_{3},
$$

a contradiction. Therefore there can be at most one concurrent pair among the pairs having the same ratio. Thus the number of concurrent pairs is at 
most $|W(U)|$. Hence

$$
\sum_{i \geq 2}\left(\begin{array}{l}
i \\
2
\end{array}\right) s_{i} \leq|W(U)|
$$

This proves the lemma.

We now specialize $R$ to be the set of $a_{i}$ 's. Under condition (2.2) or (2.3) we see from Lemma 2.2 that $R$ has property $P_{2}$ or $P_{3}$. We apply Lemmas 4.1 and 4.2 to show

Lemma 4.5. Let $m, i$ and $T$ be given positive integers. Suppose the $a_{j}$ 's are not divisible by $p_{i}$ and are arranged in the increasing order as

$$
b_{1}<b_{2}<\cdots .
$$

Suppose further that the $a_{j}$ 's have property $P_{2}$. Assume that $(U, V)$ is a multiplicative covering for the set $S$ of all integers in $\left[1, b_{h}\right]$ not divisible by $p_{i}$ as constructed in Lemma 4.1. Then

$$
b_{h} \geq \alpha(h-\beta)
$$

where

$$
\alpha^{-1}=\sum_{j=1, j \neq i}^{m+1} \frac{\varphi\left(p_{1} \cdots p_{j-1} p_{i}^{(j)}\right)}{p_{1} \cdots p_{j-1} p_{i}^{(j)} r_{j}(T)}, \quad \beta=|W(U)|+\sum_{j=1, j \neq i}^{m+1} E_{j} .
$$

Proof. Let $R$ be the set of $b_{i}$ 's. Then the number of $b_{i}$ 's less than or equal to $b_{h}$ is $h$. This number does not exceed the number of edges in $G_{R}$, since $(U, V)$ is a multiplicative covering for $S$. Thus by Lemma 4.2,

$$
h \leq|V|+|W(U)|
$$

Now the result follows from Lemma 4.1 with $X=b_{h}$.

We apply Lemma 4.5 when $2,3,5$ or 7 divides $d$. Recall $\operatorname{gcd}(n, d)=1$.

Lemma 4.6. Let (1.1) hold. Suppose that the $b_{h}$ 's have property $P_{2}$.

(i) Let $2 \mid d$. Then (4.2) holds with

$$
(\alpha, \beta)=(2.571,2.17),(2.842,3.17),(3.253,7.1),(3.349,8.1) .
$$

(ii) Let $p(d)=3$. Then (4.2) holds with

$(\alpha, \beta)=(2.4,3.34),(2.666,4.34),(2.823,5.34),(2.909,6.34),(2.953,7.34)$.

(iii) Let $p(d)=5$. Then (4.2) holds with

$$
\begin{aligned}
(\alpha, \beta)= & (1.666,3.6),(2,4.6),(2.222,5.6),(2.352,6.6),(2.769,10.54), \\
& (3.185,18.54),(3.262,20.54),(3.534,36) .
\end{aligned}
$$

(iv) Let $p(d)=7$. Then (4.2) holds with

$$
\begin{aligned}
(\alpha, \beta)= & (1.867,3.27),(2.074,4.72),(2.196,5.72),(2.263,6.72), \\
& (2.584,10.86),(2.973,18.86),(3.407,38.52) .
\end{aligned}
$$


Proof. We need only specify the parameters $m$ and $T$. Then $U$ is the set of positive integers composed of $p_{1}, \ldots, p_{i-1}, p_{i+1}, \ldots, p_{m}$ and $V$ is constructed as in Lemma 4.1. The numbers $\alpha, \beta$ are computed from Lemma 4.5.

(i) Let $2 \mid d$. Take $i=1$ and $(m, T)=(2,9),(2,27),(3,15),(3,25)$, respectively.

(ii) Let $p(d)=3$. Take $i=2$ and $(m, T)=(1,8),(1,16),(1,32),(1,64)$, $(1,128)$, respectively.

(iii) Let $p(d)=5$. Take $i=3$ and $(m, T)=(1,8),(1,16),(1,32),(1,64)$, $(2,9),(2,18),(2,27),(4,21)$, respectively.

(iv) Let $p(d)=7$. Take $i=4$ and $(m, T)=(1,8),(1,16),(1,32),(1,64)$, $(2,9),(2,18),(3,18)$, respectively.

5. Application of Lemma 2.1. Inequality (2.1) proves to be basic in the problems of perfect powers in arithmetic progression, as is evident from the papers [Sa97], [SS01] and several other papers by Laishram, Mukhopadhyaya and Shorey. We refer to the survey article [Sh06] of Shorey for these references. We apply in (2.1) the lower estimates for $b_{h}$ obtained in Lemma 4.6 to get

Lemma 5.1. Suppose (1.1) holds with (2.2).

(i) The case $p(d)=2$ cannot occur.

(ii) Let $p(d)=3$. Then $k \leq 124$.

(iii) Let $p(d)=5$. Then $k \leq 374$.

(iv) Let $p(d)=7$. Then $k \leq 538$.

Proof. We see from Lemma 2.1 that

$$
\left|S_{1}\right| \geq k-\pi_{d}(k)
$$

Since the $a_{i}$ 's satisfy $P_{1}$, we get

$$
\prod_{a_{i} \in S_{1}} a_{i} \geq \prod_{i=1}^{k-\pi_{d}(k)} b_{i} .
$$

Hence, by Lemma 2.1,

$$
\prod_{i=1}^{k-\pi_{d}(k)} b_{i} \leq(k-1) ! \prod_{p \mid d} p^{-\operatorname{ord}_{p}(k-1) !} .
$$

(i) Let $2 \mid d$. Then

$$
\prod_{i=1}^{k-\pi(k)+1} b_{i} \leq \prod_{i=1}^{k-\pi_{d}(k)} b_{i} \leq(k-1) ! / 2^{\operatorname{ord}_{2}(k-1) !} .
$$


Put

$$
\delta_{h}= \begin{cases}2 h-1 & \text { for } h \leq 8 \\ 2.571(h-2.17) & \text { for } 9 \leq h \leq 12, \\ 2.842(h-3.17) & \text { for } 13 \leq h \leq 34 \\ 3.253(h-7.1) & \text { for } 35 \leq h \leq 41 \\ 3.349(h-8.1) & \text { for } h \geq 42 .\end{cases}
$$

Then by Lemma 4.6(i) we get, for every $k$,

$$
\prod_{h=1}^{k-\pi(k)+1} \delta_{h} \leq(k-1) ! / 2^{\operatorname{ord}_{2}(k-1) !} .
$$

As is standard now, we first bound $k$ using approximate values of $\pi(k)$ and $(k-1)$ !. For the remaining finite number of values of $k$, we check that the above inequality is not valid.

The proofs for (ii), (iii), and (iv) are similar. For the initial values of $\delta_{h}$ we take the $h$ th positive integer not divisible by $p_{i}$. For the other values of $h$ we choose the largest values of $\alpha(h-\beta)$ for $(\alpha, \beta)$ given in Lemma 4.6(ii), (iii), (iv), respectively.

6. Proof of the theorem. (i) Let $2 \mid d$. Then the assertion follows immediately from Lemma 4.6(i).

Now suppose $p(d)=3$. By Lemma 5.1(ii) we obtain $k \leq 124$. We apply (2.4) with $m=3, q_{1}=2, q_{2}=5, q_{3}=7, \alpha_{1}=4, \alpha_{2}=\alpha_{3}=1, h=1$, $r_{1}=5 \cdot 7$, i.e., we estimate from below the number of $a_{i}$ 's composed of 2,5 and 7 with their powers not exceeding 4, 1, 1 and not divisible by 35 . This yields

$$
C(k, 3,4,1,1,5 \cdot 7) \geq 8 \text { for } 16 \leq k \leq 124 .
$$

For any $k$, we denote by $S(k)=S\left(k, \beta_{1}, \ldots, \beta_{n_{1}}, X\right)$ the set of $a_{i}$ 's $\subseteq Y$ where $X, Y, \beta_{1}, \ldots, \beta_{n_{1}}$ are as in Lemma 3.1. In the notation of Lemma 3.1, we take $X=\left\{1,2,2^{2}, 2^{3}, 2^{4}\right\}$, with $r=4$ and $n_{1}=3,\left\{\beta_{1}, \beta_{2}, \beta_{3}\right\}=\{1,5,7\}$. By (6.1), we get

$$
|S(k)| \geq 8>n_{1}+r
$$

a contradiction to Lemma 3.1 .

Now we consider $4 \leq k \leq 15$. We take $m=1, q_{1}=2, \alpha_{1}=2, h=0$ to find

$$
C(k, 1,2) \geq 3 \text {. }
$$

This means that there are at least three $a_{i}$ 's belonging to $\left\{1,2,2^{2}\right\}$. Since $a_{i}$ 's are distinct this means property $P_{2}$ is not satisfied.

(ii) Let $p(d)=5$. By Lemma 5.1(iii), we have $k \leq 374$. 
Let $65 \leq k \leq 374$. Take $X=\left\{1,2,2^{2}, 2^{3}, 2^{4}, 2^{5}\right\}, n_{1}=15$,

$$
\begin{aligned}
\left\{\beta_{1}, \ldots, \beta_{15}\right\}=\{1,3,7,11,13,3 \cdot 7,3 \cdot 11,3 \cdot 13,7 \cdot 11,7 \cdot 13, & \left.11 \cdot 13,3^{2}, 3^{2} \cdot 7,3^{2} \cdot 11,3^{2} \cdot 13\right\} .
\end{aligned}
$$

We apply (2.4) with $m=5, q_{1}=2, q_{2}=3, q_{3}=7, q_{4}=11, q_{5}=13, \alpha_{1}=5$, $\alpha_{2}=2, \alpha_{3}=\alpha_{4}=\alpha_{5}=1, h=4, r_{1}=3 \cdot 7 \cdot 11, r_{2}=3 \cdot 7 \cdot 13, r_{3}=3 \cdot 11 \cdot 13$, $r_{4}=7 \cdot 11 \cdot 13$ to get

$$
|S(k)| \geq 21 \text {. }
$$

This contradicts Lemma 3.1 with $r=5$.

For $25 \leq k \leq 64$, take $X=\left\{1,2,2^{2}, 2^{3}, 2^{4}\right\}, n_{1}=4,\left\{\beta_{1}, \beta_{2}, \beta_{3}, \beta_{4}\right\}=$ $\left\{1,3,3^{2}, 7\right\}$. Apply (2.4) with $m=3, q_{1}=2, q_{2}=3, q_{3}=7, \alpha_{1}=4, \alpha_{2}=2$, $\alpha_{3}=1, h=1, r_{1}=3 \cdot 7$ to get

$$
|S(k)| \geq 9,
$$

contradicting Lemma 3.1 with $r=4$.

Let $9 \leq k \leq 24$. Take $X=\left\{1,2,2^{2}, 2^{3}\right\}, n_{1}=2,\left\{\beta_{1}, \beta_{2}\right\}=\{1,3\}$. Apply (2.4) with $m=2, q_{1}=2, q_{2}=3, \alpha_{1}=3, \alpha_{2}=1, h=0$ to get

$$
|S(k)| \geq 5,
$$

except for $k=19,20,23,24$ in which cases $|S(k)| \geq 4$. By Lemma 3.1, we have $|S(k)| \leq 4\left(=2 n_{1}\right)$. Thus we need to consider $k=19,20,23,24$ with $|S(k)|$ $=4$. Let $k=24$. Then 23 divides $a_{0}, a_{23} ; 7$ divides $a_{1}, a_{8}, a_{15}, a_{22} ; 19$ divides $a_{2}, a_{21}$; and 17 divides $a_{3}, a_{20}$. Then 16 divides one of $a_{0}, a_{1}, a_{2}, a_{3}, a_{20}, a_{21}$, $a_{22}, a_{23}$. Thus the number of $a_{i}$ 's divisible by 16 and not by the primes 7 , 17,19 and 23 is at most 1 . Hence $|S(k)| \geq 5$, a contradiction. We give for other values of $k$ the combination of $a_{i}$ 's divisible by certain primes or 16 or 9 , by which $|S(k)| \geq 5$, to get a contradiction.

- $k=23$ : 11 divides $a_{0}, a_{11}, a_{22}$, but no distinct placings for 4 multiples of 7 .

- $k=20$ : 19 divides $a_{0}, a_{19} ; 17$ divides $a_{1}, a_{18}$; no place for 2 multiples of 16 .

- $k=19: 9$ divides $a_{0}, a_{9}, a_{18}$, no place for 2 multiples of 17 .

This proves that $D_{1}>E_{1}$ if $k \geq 9$.

Let $k=6$. There are at most three multiples of 2 and two multiples of 3 among the $a_{i}$ 's, but they cannot be distinct. Hence at least two $a_{i}$ 's are equal to 1 .

Let $k=8$. If there are two multiples of 7 , then 7 divides $a_{0}$ and $a_{7}$ and we can apply the case $k=6$ to $a_{1}, \ldots, a_{6}$. Otherwise there is at most one multiple of 7 , of 8 , and of 9 . Hence there are at least five $a_{i}$ 's with values in $\{1,2,4,3,6,12\}$. But the $a_{i}$ 's are distinct and they cannot assume all the three values from either $\{1,2,4\}$ or $\{3,6,12\}$. This yields a contradiction. 
Let $k=7$. There is at most one multiple of 7 , one multiple of 8 and one multiple of 9 . Hence there are at least four $a_{i}$ 's in $\{1,2,3,4,6,12\}$. A simple check shows that this cannot happen if $P_{3}$ holds.

(iii) Let $p(d)=7$. By Lemma 5.1 (iv), we have $k \leq 538$. As seen in the case $5 \mid d$, we will be applying (2.4) and Lemmas 3.1 and 3.2 with suitable choices of parameters for various ranges of values of $k$ so that the lower bound for $C\left(k, m, \alpha_{1}, \ldots, \alpha_{m}, r_{1}, \ldots, r_{h}\right)$ and the upper bound for $|S(k)|$ contradict each other. We give below the range of $k$ and the choice of the parameters.

(a) $118 \leq k \leq 538$ : By (2.4) we have

$$
C\left(k, 5,5,4,2,1,1,3 \cdot 5 \cdot 11,3^{2} 5^{2}, 3 \cdot 5 \cdot 13,3 \cdot 11 \cdot 13\right) \geq 35 .
$$

Now take $X=\left\{1,2,2^{2}, 2^{3}, 2^{4}, 2^{5}\right\}, Z=\left\{1,3,3^{2}, 3^{3}, 3^{4}\right\}, n_{1}=29,\left\{\beta_{1}, \ldots, \beta_{29}\right\}$ $=\left\{Z, 5 Z, 5^{2}, 3 \cdot 5^{2}, 11 Z, 5 \cdot 11,5^{2} 11,13 Z, 5 \cdot 13,5^{2} 13,11 \cdot 13,5 \cdot 11 \cdot 13,5^{2} \cdot 11 \cdot 13\right\}$ to get

$$
|S(k)| \leq 29+5=34,
$$

by Lemma 3.1, which gives the necessary contradiction.

(b) $36 \leq k \leq 117$ : By $(2.4)$ we have $C(k, 3,4,3,1) \geq 13$. Now take $X=\left\{1,2,2^{2}, 2^{3}, 2^{4}\right\}, Z=\left\{1,3,3^{2}, 3^{3}\right\}, n_{1}=8,\left\{\beta_{1}, \ldots, \beta_{8}\right\}=\{Z, 5 Z\}$. Thus $|S(k)| \leq 8+4=12$, by Lemma 3.1 , which gives a contradiction.

(c) $25 \leq k \leq 35$ : By $(2.4)$ we have $C(k, 3,3,2,1) \geq 10$. Now take $X=$ $\left\{1,2,2^{2}, 2^{3}\right\}, Z=\left\{1,3,3^{2}\right\}, n_{1}=6,\left\{\beta_{1}, \ldots, \beta_{6}\right\}=\{Z, 5 Z\}$. Thus $|S(k)| \leq$ $6+3=9$, by Lemma 3.1, which gives a contradiction.

(d) $15 \leq k \leq 24$ : By $(2.4)$ we have $C(k, 2,4,2) \geq 6$. Now take $X=$ $\left\{1,2,2^{2}, 2^{3}, 2^{4}\right\}, Z=\left\{1,3,3^{2}\right\}, n_{1}=3,\left\{\beta_{1}, \beta_{2}, \beta_{3}\right\}=\{Z\}$. Thus $|S(k)| \leq 5$, by Lemma 3.2 , which gives a contradiction.

(e) $8 \leq k \leq 14$ : By (2.4) we have $C(k, 2,2,1) \geq 4$ if $k=8,9,10$ and $C(k, 2,2,1) \geq 3$ if $11 \leq k \leq 14$. Using the argument as in the case $5 \mid d$, $k \in\{19,20,23,24\}$, we can improve this as

$$
C(k, 2,2,1) \geq 4 \quad \text { if } 11 \leq k \leq 14 .
$$

Suppose $C(k, 2,2,1)=3$. We give the combination of $a_{i}$ 's divisible by certain primes or 8 or 9 which shows that there is a coincidence among the $a_{i}$ 's.

- $k=14$ : 13 divides $a_{0}, a_{13} ; 11$ divides $a_{1}, a_{12}$; no place for 3 multiples of 5 .

- $k=13: 11$ divides $a_{0}, a_{11} ; 5$ divides $a_{2}, a_{7}, a_{12} ; 9$ divides $a_{1}, a_{10}$; or 11 divides $a_{1}, a_{12} ; 5$ divides $a_{0}, a_{5}, a_{10} ; 9$ divides $a_{2}, a_{11}$; in both cases no place for 2 multiples of 8 .

- $k=12: 11$ divides $a_{0}, a_{11}$; no place for 3 multiples of 5 .

- $k=11$ : 5 divides $a_{0}, a_{5}, a_{10}$; no place for 2 multiples of 9 . 
Thus for $8 \leq k \leq 14$,

$$
|S(k)| \geq 4
$$

a contradiction to Lemma 3.2.

\section{References}

[BBGH06] M. A. Bennett, N. Bruin, K. Győry and L. Hajdu, Powers from products of consecutive terms in arithmetic progression, Proc. London Math. Soc. (3) 92 (2006), 273-306.

[ES75] P. Erdös and J. L. Selfridge, The product of consecutive integers is never a power, Illinois J. Math. 19 (1975), 292-301.

[G99] K. Györy, Power values of products of consecutive integers and binomial coefficients, in: Number Theory and its Applications, S. Kanemitsu and K. Györy (eds.), Kluwer, 1999, 145-156.

[Sa97] N. Saradha, On perfect powers in products with terms from arithmetic progressions, Acta Arith. 82 (1997), 147-172.

[SS01] N. Saradha and T. N. Shorey, Almost perfect powers in arithmetic progression, ibid. 99 (2001), 363-388.

[SS05] - - Contributions towards a conjecture of Erdös on perfect powers in arithmetic progression, Compos. Math. 141 (2005), 541-560.

[Sh88] T. N. Shorey, Some exponential diophantine equations, in: New Advances in Transcendence Theory, A. Baker (ed.), Cambridge Univ. Press, 1988, 352365.

[Sh06] -, Diophantine approximations, Diophantine equations, transcendence and applications, Indian J. Pure Appl. Math. 37 (2006), 9-39.

[ST90] T. N. Shorey and R. Tijdeman, Perfect powers in products of terms in an arithmetical progression, Compos. Math. 75 (1990), 307-344.

School of Mathematics

Tata Institute of Fundamental Research

Homi Bhabha Road

Mumbai 400005, India

E-mail: saradha@math.tifr.res.in
Mathematical Institute

Leiden University P.O. Box 9512

2300 RA Leiden, Netherlands

E-mail: tijdeman@math.leidenuniv.nl

Received on 24.8.2007

and in revised form on 12.12.2007 